\title{
Diameter three orientability of bipartite graphs
}

\author{
Bin Chen An Chang* \\ Fuzhou University \\ Fuzhou, Fujian, P.R. China \\ cbfzu03@163.com anchang@fu.edu.cn
}

Submitted: Jul 17, 2020; Accepted: Apr 26, 2021; Published: May 21, 2021

(C) The authors. Released under the CC BY-ND license (International 4.0).

\begin{abstract}
In 2019, Czabarka, Dankelmann and Székely showed that for every undirected graph of order $n$, the minimum degree threshold for diameter two orientability is $\frac{n}{2}+\Theta(\ln n)$. In this paper, we consider bipartite graphs and give a sufficient condition in terms of the minimum degree for such graphs to have oriented diameter three. We in particular prove that for balanced bipartite graphs of order $n$, the minimum degree threshold for diameter three orientability is $\frac{n}{4}+\Theta(\ln n)$.
\end{abstract}

Mathematics Subject Classifications: 05C12, 05C20

\section{Introduction}

We will use the notation from [1]. All graphs and digraphs considered here have no loops or parallel edges/arcs. Let $G=(V, E)$ be an undirected graph with vertex set $V=V(G)$ and edge set $E=E(G)$. The order (size) of $G$ is the number of vertices (edges) in $G$. We write $e(G)$ for the size of $G$. Let $N_{G}(v)$ be the subset of vertices incident with $v$, and the degree of $v$ is $d_{G}(v)=\left|N_{G}(v)\right|$. We denote by $\delta(G)$ and $\Delta(G)$ the minimum and maximum degrees of the vertices of $G$. Let $D=(V, A)$ denote a digraph, where $V=V(D)$ is its vertex set and $A=A(D)$ its arc set. For an $\operatorname{arc}(u, v) \in A(D)$, the vertex $u$ is its tail and the second vertex $v$ is its head. For a vertex $v$ in $D$, the out-neighbourhood (in-neighbourhood, respectively) of $v$ is the vertex set $N_{D}^{+}(v)=\{u \in$ $V-v: v u \in A\}\left(N_{D}^{-}(v)=\{u \in V-v: u v \in A\}\right.$, respectively). The out-degree (in-degree, respectively) of $v$ is $d_{D}^{+}(v)=\left|N_{D}^{+}(v)\right|\left(d_{D}^{-}(v)=\left|N_{D}^{-}(v)\right|\right.$, respectively).

A path of length $\ell$ of $G$ is a list $v_{0}, v_{1}, \ldots, v_{\ell}$ of distinct vertices such that $\left(v_{i}, v_{i+1}\right) \in$ $E(G)$ for $0 \leqslant i \leqslant \ell-1$. An undirected graph is connected, if it contains an $(u, v)$-path for every pair $u, v$ of its vertices. For digraphs, a directed path of length $\ell$ of $D$ is a list $v_{0}, v_{1}, \ldots, v_{\ell}$ of distinct vertices such that $\left(v_{i}, v_{i+1}\right) \in A(D)$ for $0 \leqslant i \leqslant \ell-1$. Such a

\footnotetext{
${ }^{*}$ Supported by the National Natural Science Foundation of China (Grant No. 11471077)
} 
directed path of length $\ell$ is called directed $\ell$-path. A digraph $D$ is strong if and only if for any $u, v \in V(D)$, there is a directed path from $u$ to $v$.

In an undirected graph $G$, the distance from $u$ to $v$ is the number of edges in a shortest path from $u$ to $v$. We denote the distance from $u$ to $v$ by dist $(u, v)$. The diameter of an undirected graph $G$ is the maximum distance between two vertices. The definition for digraphs is analogous. We write $\operatorname{diam}(G)$ and $\operatorname{diam}(D)$ for the diameter of an undirected graph $G$ and a digraph $D$, respectively.

A bridge is an edge of a connected graph whose removal renders the graph disconnected. We call an undirected graph bridgeless, if it is connected and contains no bridge. An orientation $D$ of an undirected graph $G$ is a digraph on the same vertex set $V(G)$ obtained by assigning every edge of $G$ an orientation. An orientation $D$ of $G$ is strong, if every two vertices in $D$ are mutually reachable in $D$. The oriented diameter $\overrightarrow{\operatorname{diam}}(G)$ of $G$ is the minimum diameter among all strong orientations of $G$.

A well-known result, due to Robbins [15], states that every bridgeless graph has a strong orientation. This theorem was inspired by an application in traffic control, to make traffic flow more efficient by use of one-way streets, to be precise. It is natural to seek an orientation whose diameter is as small as possible.

As early as 1978, Chvátal and Thomassen [4] proved that there exists a function $f$ such that every bridgeless graph of diameter $d$ has an oriented diameter at most $f(d)$. Furthermore, they proved that $f(2)=6$ and $\frac{1}{2} d^{2}+d \leqslant f(d) \leqslant 2 d^{2}+2 d$, the lower bound is acquired by constructing a family of graphs, while their construction is not correct for odd $d$. Recently, Kwok, Liu and West [12] fixed the construction for odd $d$ and also showed that $9 \leqslant f(3) \leqslant 11$.

Since then, several upper bounds on $\overrightarrow{\operatorname{diam}}(G)$ in terms of certain graph invariants such as the domination number $[8,11]$, the maximum degree $[7]$ and the minimum degree $[2,18]$ have been given. For a survey on the numerous further results on the oriented diameter published over the past decades, see, e.g., Koh and Tay [10].

For any bridgeless graph $G$, it is obvious that $\overrightarrow{\operatorname{diam}}(G) \geqslant 2$. As expected, complete graphs are extremal graphs except for one singular case.

Theorem 1. (Plesnik [14], Boesch and Tindell [3], Maurer [13]) For $n \geqslant 3$,

$$
\overrightarrow{\operatorname{diam}}\left(K_{n}\right)= \begin{cases}2, & \text { if } n \neq 4, \\ 3, & \text { if } n=4 .\end{cases}
$$

Šoltés [17], and independently Gutin [9] obtained a comprehensive result on complete bipartite graphs.

Theorem 2. (Šoltés [17], Gutin [9]) For $q \geqslant p \geqslant 2$,

$$
\overrightarrow{\operatorname{diam}}\left(K_{p, q}\right)= \begin{cases}3, & \text { if } q \leqslant\left(\begin{array}{c}
p \\
\left\lfloor\frac{p}{2}\right\rfloor
\end{array}\right), \\
4, & \text { if } q>\left(\begin{array}{c}
p \\
\left\lfloor\frac{p}{2}\right\rfloor
\end{array}\right) .\end{cases}
$$


In 2018, Dankelmann, Guo and Surmacs [7] considered the oriented diameter of a bridgeless graph $G$ of order $n$ in terms of the maximum degree. They proved the upper bound $\overrightarrow{\operatorname{diam}}(G) \leqslant n-\Delta(G)+3$. Moreover, they constructed an infinite family of undirected graphs whose oriented diameter is equal to this upper bound. Furthermore, they showed that, if $G$ is bipartite, the bound above can be improved:

Theorem 3. (Dankelmann, Guo and Surmacs [7]) Let $G$ be a bridgeless bipartite graph with partite sets $X$ and $Y, v \in Y$. Then,

$$
\overrightarrow{\operatorname{diam}}(G) \leqslant 2\left(|X|-d_{G}(v)\right)+7 .
$$

For a bridgeless balanced bipartite graph, that is, a bipartite graph whose partite sets have the same cardinality, by Theorem 3, a straightforward result is the following:

Corollary 4. [7] Let $G$ be a bridgeless balanced bipartite graph of order $n$ and maximum degree $\Delta(G)$. Then,

$$
\overrightarrow{\operatorname{diam}}(G) \leqslant n-2 \Delta(G)+7
$$

Meanwhile, they constructed an infinite family of bridgeless balanced bipartite graphs of order $n$ whose oriented diameter reaches this upper bound.

In 2019, Czabarka, Dankelmann and Székely [6] proved the following theorem:

Theorem 5. (Czabarka, Dankelmann and Székely [6]) Let $G$ be an undirected graph on $n$ vertices. If

$$
\delta(G) \geqslant \frac{n}{2}+\frac{\ln n}{\ln \left(\frac{4}{3}\right)}=\frac{1}{2} n+(3.476 \ldots) \ln n,
$$

then $\overrightarrow{\operatorname{diam}}(G)=2$.

Furthermore, they constructed an infinite family of undirected graphs $G$ of order $n$ with $\delta(G) \geqslant \frac{n}{2}+\frac{\ln n}{2 \ln \left(\frac{27}{4}\right)}=\frac{1}{2} n+(0.261 \ldots) \ln n$ whose oriented diameter is more than two.

Motivated by the work cited above, we consider the oriented diameter of bipartite graphs. Let $\kappa \geqslant 1$ be a positive integer and consider bipartite graphs with partite sets $X$ and $Y$, such that $|Y|=\kappa|X|+\gamma$ and $0 \leqslant \gamma<|X|$. For large enough $X$, we give a sufficient condition in terms of minimum degrees for vertices in $X$ and $Y$, respectively, for the diameter three orientability of the bipartite graph $G$. Furthermore, we construct an infinite family of bipartite graphs with oriented diameter at least four, which have almost as big minimum degrees in the partite sets as the bound in our sufficient condition. Specialising our results for balanced bipartite graphs of order $n$, in which $|X|=|Y|=\frac{n}{2}$, the minimum degree threshold for diameter three orientability is $\frac{n}{4}+\Theta(\ln n)$. 


\section{Main results}

We give a sufficient condition by means of a proof technique which is known as the probabilistic method. Roughly speaking, this technique is based on an understanding of how graphs behave on the average, i.e., whether there exists some desired property or not. In a probability space $(\Omega, P)$, any subset $A$ of $\Omega$ is referred to as an event. Denote by $P[A]$ the probability of the event $A$.

Let $G$ be a bipartite graph with partite sets $X$ and $Y$, we denote $\delta(X)=\min \left\{d_{G}(x)\right.$ : $x \in X\}$ and $\delta(Y)=\min \left\{d_{G}(y): y \in Y\right\}$, respectively.

Theorem 6. Let $\kappa \geqslant 1$ be a positive integer and let $G$ be a bipartite graph of order $n$ with partite sets $X$ and $Y$ which satisfies that $|X|$ is sufficiently large and $|Y|=\kappa|X|+\gamma$, where $0 \leqslant \gamma<|X|$. If

$$
\delta(X) \geqslant \frac{|Y|}{2}+\frac{\ln ((\kappa+2)|Y|)}{\ln \left(\frac{8}{7}\right)} \text { and } \delta(Y) \geqslant \frac{|X|}{2}+\frac{\ln ((\kappa+2)|X|)}{\ln \left(\frac{8}{7}\right)},
$$

then $\overrightarrow{\operatorname{diam}}(G)=3$.

Proof. Define $f: \mathbb{N} \rightarrow \mathbb{R}, n \mapsto \frac{\ln ((\kappa+2) n)}{\ln \left(\frac{8}{7}\right)}, p=|X|$, and $q=|Y|$. It is clear that $p+q=n$ and $p \leqslant q<(\kappa+1) p$. Assume that the minimum degree of vertex in $X$ is at least $q / 2+f(q)$ and the minimum degree of vertex in $Y$ is at least $p / 2+f(p)$. Obviously, we have $f(q) \geqslant f(p), f(p) \leqslant p / 2$ and $f(q) \leqslant q / 2$.

Since $p$ is sufficiently large, so we have $q \leqslant\left(\begin{array}{c}p \\ \left\lfloor\frac{p}{2}\right\rfloor\end{array}\right)$. By Theorem $2, \overrightarrow{\operatorname{diam}}(G) \geqslant$ $\overrightarrow{\operatorname{diam}}\left(K_{p, q}\right)=3$ since $G$ is a subgraph of $K_{p, q}$, implying that if we select large enough $f(p)$ and $f(q), \overrightarrow{\operatorname{diam}}(G)$ can be 3 .

We orient every edge of $E(G)$ randomly and independently with probability $1 / 2$. Let $u, v$ be two vertices of $V(G)$ and define the random variable $A_{u v}$ to be 1 if there is no directed $\ell$-path from $u$ to $v$, where $\ell \leqslant 3$, and 0 if there is such a directed path.

Let $\eta$ denote the expected number of ordered pairs $(u, v)$ where no directed path of length at most 3 exists from $u$ to $v$. Hence, we have $\eta=\sum_{u, v \in V(G), u \neq v} P\left[A_{u v}\right]$.

We deal with the directed paths by distinguishing the following two cases:

Case 1. $u, v$ belong to the same partite set.

Subcase 1. $u, v \in X$.

Since $G$ is bipartite, we have $N_{G}(u) \subseteq Y$ and $N_{G}(v) \subseteq Y$. Then, by the inclusionexclusion formula, the following holds:

$$
\left|N_{G}(u) \cap N_{G}(v)\right|=\left|N_{G}(u)\right|+\left|N_{G}(v)\right|-\left|N_{G}(u) \cup N_{G}(v)\right| \geqslant 2 f(q) .
$$

Hence, the expected value of $A_{u v}$ is $P\left[A_{u v}=1\right]=\left(\frac{3}{4}\right)^{\left|N_{G}(u) \cap N_{G}(v)\right|} \leqslant\left(\frac{3}{4}\right)^{2 f(q)}$.

Subcase 2. $u, v \in Y$.

Similarly, $N_{G}(u) \subseteq X$ and $N_{G}(v) \subseteq X$, we have:

$$
\left|N_{G}(u) \cap N_{G}(v)\right|=\left|N_{G}(u)\right|+\left|N_{G}(v)\right|-\left|N_{G}(u) \cup N_{G}(v)\right| \geqslant 2 f(p) .
$$


Thus, we obtain that $P\left[A_{u v}=1\right]=\left(\frac{3}{4}\right)^{\left|N_{G}(u) \cap N_{G}(v)\right|} \leqslant\left(\frac{3}{4}\right)^{2 f(p)}$.

Case 2. $u, v$ belong to different partite sets. Without loss of generality, we may assume that $u \in X$ and $v \in Y$.

Subcase 1. $u, v$ are adjacent in $G$.

Obviously, $N_{G}(u) \subseteq Y$. For each $w \in N_{G}(u)$, we have $\left|N_{G}(v) \cap N_{G}(w)\right| \geqslant 2 f(p)$. It is clear that there exist at least $2 f(p)-1$ edge-disjoint $(u, v)$-paths in $G$ whose length is no more than three. Thus, we obtain that $P\left[A_{u v}=1\right] \leqslant \frac{1}{2}\left(\frac{7}{8}\right)^{2 f(p)-1}<\left(\frac{7}{8}\right)^{2 f(p)}$.

Subcase 2. $u, v$ are not adjacent in $G$.

Similar to Subcase 1 , for each $w \in N_{G}(u)$ there exist at least $2 f(p)$ edge-disjoint $(u, v)$ paths in $G$ whose length is no more than three. Hence, we have $P\left[A_{u v}=1\right] \leqslant\left(\frac{7}{8}\right)^{2 f(p)}$.

Therefore, the following holds:

$$
\begin{aligned}
\sum_{u, v \in V(G), u \neq v} P\left[A_{u v}\right] & \leqslant 2\left(\begin{array}{l}
p \\
2
\end{array}\right)\left(\frac{3}{4}\right)^{2 f(q)}+2\left(\begin{array}{l}
q \\
2
\end{array}\right)\left(\frac{3}{4}\right)^{2 f(p)}+2 p q\left(\frac{7}{8}\right)^{2 f(p)} \\
& <p^{2}\left(\frac{3}{4}\right)^{2 f(p)}+q^{2}\left(\frac{3}{4}\right)^{2 f(p)}+2 p q\left(\frac{7}{8}\right)^{2 f(p)} \\
& <p^{2}\left(\frac{7}{8}\right)^{2 f(p)}+(\kappa+1)^{2} p^{2}\left(\frac{7}{8}\right)^{2 f(p)}+2(\kappa+1) p^{2}\left(\frac{7}{8}\right)^{2 f(p)} \\
& =(\kappa+2)^{2} p^{2}\left(\frac{7}{8}\right)^{2 f(p)} \\
& =1
\end{aligned}
$$

We obtain that $\eta=\sum_{u, v \in V(G), u \neq v} P\left[A_{u v}\right]<1$, which implies that there exists at least one orientation with $\overrightarrow{\operatorname{diam}}(G) \leqslant 3$. This completes the proof of theorem.

We now construct an infinite family of bipartite graphs $G_{t}$ with large minimum degree which satisfies $\overrightarrow{\operatorname{diam}}\left(G_{t}\right) \geqslant 4$.

Definition 7. Let $X_{1}, X_{2}, X_{3}, Y_{1}, Y_{2}, Y_{3}, Y_{4}$ be disjoint vertex sets with $\left|X_{2}\right|=\left|Y_{2}\right|=3 t$, $\left|X_{1}\right|=\left|X_{3}\right|=\left|Y_{1}\right|=\left|Y_{3}\right|=\left(\begin{array}{c}3 t \\ t\end{array}\right)$ and $\left|Y_{4}\right|=(\kappa-1)\left(2\left(\begin{array}{c}3 t \\ t\end{array}\right)+3 t\right)+\gamma$, where $\kappa \geqslant 1$ is a positive integer and $0 \leqslant \gamma<2\left(\begin{array}{c}3 t \\ t\end{array}\right)+3 t$. Moreover, let $R \rightarrow u_{R}$ and $R \rightarrow v_{R}$ (also, $S \rightarrow u_{S}$, $S \rightarrow v_{S}$ ) be bijections from the $2 t$-element subsets of $X_{2}$ to $Y_{1}$ and resp., $Y_{3}$ (also from the $2 t$-element subsets of $Y_{2}$ to $X_{1}$ and resp., $X_{3}$ ). The bipartite graph $G_{t}$ is defined on bipartite sets $X=X_{1} \cup X_{2} \cup X_{3}$ and $Y=Y_{1} \cup Y_{2} \cup Y_{3} \cup Y_{4}$ by adding all edges between $X_{i}$ and $Y_{i} \cup Y_{4}$ for each $i \in\{1,2,3\}$ and for each $2 t$-element subset $R$ of $X_{2}$ (and 2t-element subset $S$ of $Y_{2}$ ) adding all edges from vertices of $R$ to $u_{R}$ and $v_{R}$ (and from vertices of $S$ to $u_{S}$ and $\left.v_{S}\right)$.

Theorem 8. $\overrightarrow{\operatorname{diam}}\left(G_{t}\right) \geqslant 4$.

Proof. Let $D_{t}$ be an arbitrary strong orientation of $G_{t}$. Let $y$ be a vertex of $Y_{1}$. Obviously, $N_{G_{t}}(y)=\left(N_{G_{t}}(y) \cap X_{1}\right) \cup\left(N_{G_{t}}(y) \cap X_{2}\right)$. Furthermore, $\left|N_{G_{t}}(y) \cap X_{2}\right|=2 t$. Hence, we 
have $\left|N_{D_{t}}^{+}(y) \cap X_{2}\right|+\left|N_{D_{t}}^{-}(y) \cap X_{2}\right|=2 t$, which implies that either $\left|N_{D_{t}}^{+}(y) \cap X_{2}\right| \leqslant t$ or $\left|N_{D_{t}}^{-}(y) \cap X_{2}\right| \leqslant t$. Without loss of generality, we may assume that $\left|N_{D_{t}}^{+}(y) \cap X_{2}\right| \leqslant$ $t$. Since $\left|X_{2}\right|=3 t$, then there exists a subset $R$ of $X_{2}$ with cardinality $2 t$ such that $R \cap\left(N_{D_{t}}^{+}(y) \cap X_{2}\right)=\emptyset$. Set $w=v_{R}$. Since $y$ and $w$ are different vertices in the same partition class $Y$, and $R \cap\left(N_{D_{t}}^{+}(y) \cap X_{2}\right)=\emptyset$, $\operatorname{dist}(y, w)$ is an even number bigger than 2 . The statement follows.

Analogously, we denote $\delta_{t}(X)=\min \left\{d_{G_{t}}(x): x \in X\right\}$ and $\delta_{t}(Y)=\min \left\{d_{G_{t}}(y): y \in\right.$ $Y\}$ in $G_{t}$.

Theorem 9. For sufficiently large $t$, we have

$$
\delta_{t}(X) \geqslant \frac{(2 \kappa-1)|Y|}{2 \kappa}+\frac{\ln |Y|}{2 \ln \frac{27}{4}} \text { and } \delta_{t}(Y) \geqslant \frac{|X|}{2}+\frac{\ln ((\kappa+1)|X|)}{2 \ln \frac{27}{4}}
$$

in $G_{t}$ as defined in Definition $\%$.

Proof. Clearly, $|X|=2\left(\begin{array}{c}3 t \\ t\end{array}\right)+3 t$ and $|Y|=\kappa\left(2\left(\begin{array}{c}3 t \\ t\end{array}\right)+3 t\right)+\gamma$. Denote by $n_{t}=\left|V\left(G_{t}\right)\right|$, we have $n_{t}=(\kappa+1)\left(2\left(\begin{array}{c}3 t \\ t\end{array}\right)+3 t\right)+\gamma<(\kappa+2)\left(2\left(\begin{array}{c}3 t \\ t\end{array}\right)+3 t\right)$.

If $u \in X_{1} \cup X_{3}$, then $d_{G_{t}}(u)=\left(\begin{array}{c}3 t \\ t\end{array}\right)+2 t+(\kappa-1)\left(2\left(\begin{array}{c}3 t \\ t\end{array}\right)+3 t\right)+\gamma$. If $v \in X_{2}$, we have $d_{G_{t}}(v)=2\left(\begin{array}{c}3 t-1 \\ 2 t-1\end{array}\right)+3 t+(\kappa-1)\left(2\left(\begin{array}{c}3 t \\ t\end{array}\right)+3 t\right)+\gamma=\frac{4}{3}\left(\begin{array}{c}3 t \\ t\end{array}\right)+3 t+(\kappa-1)\left(2\left(\begin{array}{c}3 t \\ t\end{array}\right)+3 t\right)+\gamma$. If $w \in Y_{1} \cup Y_{3}$, then $d_{G_{t}}(w)=\left(\begin{array}{c}3 t \\ t\end{array}\right)+2 t$. If $x \in Y_{2}$, we have $d_{G_{t}}(x)=2\left(\begin{array}{c}3 t-1 \\ 2 t-1\end{array}\right)+3 t=\frac{4}{3}\left(\begin{array}{c}3 t \\ t\end{array}\right)+3 t$. If $y \in Y_{4}$, then $d_{G_{t}}(y)=2\left(\begin{array}{c}3 t \\ t\end{array}\right)+3 t$. Hence, we have $\delta_{t}(X)=\left(\begin{array}{c}3 t \\ t\end{array}\right)+2 t+(\kappa-1)\left(2\left(\begin{array}{c}3 t \\ t\end{array}\right)+3 t\right)+\gamma$ and $\delta_{t}(Y)=\left(\begin{array}{c}3 t \\ t\end{array}\right)+2 t$. Moreover, it is clear that $\delta_{t}(X)>\frac{(2 \kappa-1)|Y|}{2 \kappa}+\frac{t}{2}$ and $\delta_{t}(Y)=\frac{|X|}{2}+\frac{t}{2}$.

By the Robbins' formula [16], the following equality holds:

$$
\left(\begin{array}{c}
3 t \\
t
\end{array}\right)=\frac{(3 t) !}{t !(2 t) !}=\frac{\left(\frac{3 t}{e}\right)^{3 t} \sqrt{6 \pi t} e^{\alpha_{1}}}{\left(\frac{t}{e}\right)^{t} \sqrt{2 \pi t} e^{\alpha_{2}}\left(\frac{2 t}{e}\right)^{2 t} \sqrt{4 \pi t} e^{\alpha_{3}}}=\frac{\sqrt{3}}{2 \sqrt{\pi t}}\left(\frac{27}{4}\right)^{t} e^{\alpha_{1}-\alpha_{2}-\alpha_{3}}
$$

where $\frac{1}{36 t+1}<\alpha_{1}<\frac{1}{36 t}, \frac{1}{12 t+1}<\alpha_{2}<\frac{1}{12 t}$ and $\frac{1}{24 t+1}<\alpha_{3}<\frac{1}{24 t}$.

As $3 t \leqslant\left(\begin{array}{c}3 t \\ t\end{array}\right)$ when $t \geqslant 1$, for $c_{0}=3(\kappa+2)>0$, we have $n_{t}<(\kappa+2)\left(2\left(\begin{array}{c}3 t \\ t\end{array}\right)+3 t\right) \leqslant c_{0}\left(\begin{array}{c}3 t \\ t\end{array}\right)$. Thus, we obtain that

$$
n_{t} \leqslant c_{0}\left(\begin{array}{c}
3 t \\
t
\end{array}\right)=c_{0} \frac{\sqrt{3}}{2 \sqrt{\pi t}}\left(\frac{27}{4}\right)^{t} e^{\alpha_{1}-\alpha_{2}-\alpha_{3}} .
$$

Similarly, for sufficiently large $t$, there exists some constant $c_{1}>0$ satisfies that

$$
n_{t}<c_{1} \frac{1}{\sqrt{t}}\left(\frac{27}{4}\right)^{t}
$$

Taking logarithms of both sides of the above inequality, a simple calculation gives us that

$$
t>\frac{\ln n_{t}+\frac{1}{2} \ln t-\ln c_{1}}{\ln \frac{27}{4}}>\frac{\ln n_{t}}{\ln \frac{27}{4}} .
$$

Thus, $\delta_{t}(X)>\frac{(2 \kappa-1)|Y|}{2 \kappa}+\frac{\ln n_{t}}{2 \ln \frac{27}{4}} \geqslant \frac{(2 \kappa-1)|Y|}{2 \kappa}+\frac{\ln |Y|}{2 \ln \frac{27}{4}}$ and $\delta_{t}(Y)>\frac{|X|}{2}+\frac{\ln n_{t}}{2 \ln \frac{27}{4}} \geqslant \frac{|X|}{2}+$ $\frac{\ln ((\kappa+1)|X|)}{2 \ln \frac{27}{4}}$, as desired. 
We now consider balanced bipartite graphs of order $n$, where $n \geqslant 4$ is even, and prove a straightforward corollary.

Corollary 10. Let $\delta_{n}$ be the smallest value such that the oriented diameter of every balanced bipartite graph of order $n$ with minimum degree $\delta_{n}$ equals to three. Then

$$
\delta_{n}=\frac{n}{4}+\Theta(\ln n)
$$

Proof. Similar to the proof of Theorem 6 , let $\kappa=1$ and $\gamma=0$, it is not difficult to verify that if $\delta(G) \geqslant \frac{n}{4}+\frac{\ln n}{\ln \left(\frac{8}{7}\right)}=\frac{1}{4} n+(7.488 \ldots) \ln n$, then $\overrightarrow{\operatorname{diam}}(G)=3$. Let $G^{*}$ be a bipartite graph obtained from $G_{t}$ by deleting the vertex set $Y_{4}$. Obviously, $G^{*}$ is a balanced bipartite graph with partite sets $X=X_{1} \cup X_{2} \cup X_{3}$ and $Y=Y_{1} \cup Y_{2} \cup Y_{3}$, where $n=4\left(\begin{array}{c}3 t \\ t\end{array}\right)+6 t$. Analogously, for large $t$, we can acquire that $\overrightarrow{\operatorname{diam}}\left(G^{*}\right) \geqslant 4$ and $\delta\left(G^{*}\right) \geqslant \frac{n}{4}+\frac{\ln n}{2 \ln \left(\frac{27}{4}\right)}=\frac{1}{4} n+(0.261 \ldots) \ln n$. Thus, the corollary follows.

\section{Problem}

Koh and Tay [10] conjectured that every undirected graph $G$ of order $n$ and size at least $\left(\begin{array}{c}n \\ 2\end{array}\right)-n+5$ has an orientation of diameter two. Recently, Cochran, Czabarka, Dankelmann and Székely [5] proved this conjecture. Similarly, we consider a bipartite graph $G$ of order $n$ and propose the following problem:

Problem 11. Let $\kappa \geqslant 1$ be a positive integer and let $G$ be a bipartite graph of order $n$ with partite sets $X$ and $Y$ which satisfies with $|Y|=\kappa|X|+\gamma$, where $0 \leqslant \gamma<|X|$. How many edges $e(G)$ do we need to ensure that such a bipartite graph with size at least $e(G)$ admits an orientation of diameter three?

Let $G$ be a bipartite graph of order $n$ with partite sets $X$ and $Y$, where $X=$ $\left\{x_{1}, x_{2}, \ldots, x_{p}\right\}$ and $Y=\left\{y_{1}, y_{2}, \ldots, y_{q}\right\}, q \geqslant p$, containing all edges between $X$ and $Y$ except $\left\{y_{1} x_{1}, y_{1} x_{2}, \ldots, y_{1} x_{p-2}, y_{2} x_{p}\right\}$. Obviously, $e(G)=p q-p+1$ and $\overrightarrow{\operatorname{diam}}(G) \geqslant 4$. Hence, we obtain that the minimum size must be no less than $p q-p+2$ to ensure that the oriented diameter of such bipartite graph of order $n$ equals three.

\section{Acknowledgements}

The authors would like to thank the anonymous referees for their helpful comments and suggestions. This work was supported by the National Natural Science Foundation of China (Grant No. 11471077).

\section{References}

[1] J. Bang-Jensen and G. Gutin. Digraphs: Theory, Algorithms and Applications. Springer-Verlag, London, 2nd edition, 2009. 
[2] S. Bau, P. Dankelmann. Diameter of orientations of graphs with given minimum degree. Eur. J. Comb., 49:126-133, 2015.

[3] F. Boesch, R. Tindell. Robbins' theorem for mixed multigraphs. Am. Math. Mon., $87: 716-719,1980$.

[4] V. Chvátal and C. Thomassen. Distances in orientations of graphs. J. Comb. Theory Ser. B, 24(1):61-75, 1978.

[5] G. Cochran, É. Czabarka, P. Dankelmann and A.L. Székely. A Size Condition for Diameter Two Orientable Graphs. Graphs Combin., 37:527-544, 2021.

[6] É. Czabarka, P. Dankelmann and L.A. Székely. A degree condition for diameter two orientability of graphs. Discrete Math., 342:1063-1065, 2019.

[7] P. Dankelmann, Y. Guo, M. Surmacs. Oriented diameter of graphs with given maximum degree. J. Graph Theory, 88(1):5-17, 2018.

[8] F.V. Fomin, M. Matamala, E. Prisner, and I. Rapaport. AT-free graphs: Linear bounds for the oriented diameter. Discrete Appl. Math, 141(1):135-148, 2004.

[9] G. Gutin. M-sources in complete multipartite graphs. (In Russian) Ser. Fiz.Mat.Navuk., 5(5):101-106, 1989.

[10] K.M. Koh, E.G. Tay. Optimal Orientations of Graphs and Digraphs: A Survey. Graphs Combin., 18(4):745-756, 2002.

[11] S. Kurz, M. Lätsch. Bounds for the minimum oriented diameter. Discrete Math. Theor. Comput. Sci., 14(1):109-142, 2012.

[12] P.K. Kwok, Q. Liu, and D.B. West. Oriented diameter of graphs with diameter 3. J. Combin. Theory Ser. B, 100(3):265-274, 2010.

[13] S.B. Maurer. The king chicken theorems. Math. Mag., 53:67-80, 1980.

[14] J. Plesnik. Diametrically critical tournaments. Casop. Pest. Matem., 100:361-370, 1975.

[15] H.E. Robbins. A Theorem on Graphs, with an Application to a Problem of Traffic Control. Am. Math. Mon., 46(5):281-283, 1939.

[16] H.E. Robbins. A Remark on Stirling's Formula. Am. Math. Mon., 62(1):26-29, 1955.

[17] L. Soltés. Orientations of graphs minimizing the radius of the diameter. Math. Slovaca, 36(3):289-296, 1986.

[18] M. Surmacs. Improved bound on the oriented diameter of graphs with given minimum degree. Eur. J. Comb., 59:187-191, 2017. 